I NVESTI GACI ÓN

\title{
Percepción institucional y comunitaria sobre comportamientos adictivos en Guayaquil
}

\section{Institucional and community perceptions on addictive behaviours in Guayaquil}

\author{
DrC. J usto Reinaldo Fabelo Roche,' MsC. Serguei I glesias Moré,' \\ MsC. Guillermo Díaz Llanes" " \\ I Centro de Desarrollo Académico en Drogodependencias. La Habana, Cuba. \\ "Escuela Nacional de Salud Pública. La Habana, Cuba.
}

\section{RESUMEN}

Introducción: en el mundo de hoy las conductas adictivas constituyen un grave problema para la humanidad. En la República de Ecuador la atención a pacientes consumidores de sustancias psicoactivas se ha desarrollado tradicionalmente desde la instancia privada pero actualmente se reconoce que ello ha devenido en un problema de salud que debe ser atendido por las políticas públicas.

Objetivo: identificar la percepción sobre los comportamientos adictivos en el Centro de Docencia e Investigación para el Desarrollo Humano y el Buen Vivir y sus comunidades aledañas, en Guayaquil.

Métodos: el trabajo se realizó en el 2011, con un diseño etnográfico en el que se incluyeron análisis de documentos, observación participante, entrevistas en profundidad y entrevistas grupales focalizadas. Los informantes clave se seleccionaron mediante muestreo intencional. La información obtenida fue sometida a un análisis de contenido.

Resultados: se revelaron percepciones limitadas al modelo clínico en las dimensiones de gestión comunitaria, docente-asistencial e investigativa.

Conclusiones: tanto en el Centro como en sus comunidades aledañas, la percepción sobre comportamientos adictivos está constreñida al modelo clínicoasistencial, lo que constituye una barrera para el posicionamiento del modelo salubrista.

Palabras clave: Conductas adictivas, modelo salubrista, modelo clínico, tratamiento ambulatorio, tratamiento residencial. 


\section{ABSTRACT}

Introduction: In today's world, the addictive behaviors are a serious problem for the humanity. The care of patients who consume psychoactive substances in the Republic of Ecuador has mainly been given on a private basis, but at present, it is recognized that addiction is a health problem to be managed by the public policies. Objective: to identify the perception of addictive behaviours in the Center of Education and Research for the Human Development and the Good Living and neighboring communities in Guayaquil.

Methods: the study was conducted in 2011 and adopted an ethnographic design in which document analysis, observation of participants, in-depth interviews and focused group interviews were included. The key informants were selected from an intentional sampling. The gathered information underwent a content analysis. Results: The study revealed perceptions limited to the clinical model in the community management, the teaching-assistance and the research dimensions. Conclusions: Both the Center and the neighboring communities have perceptions on addictive behaviors that are limited to the clinical-assistance model, which is a kind of barrier to the positioning of the public health model.

Key words: Addictive behaviors, public health model, clinical model, ambulatory treatment, residential treatment.

\section{NTRODUCCI ÓN}

En el mundo de hoy las conductas adictivas constituyen un grave problema para la humanidad, del que no escapa prácticamente ningún país y que afecta a millones de personas sin distinción de sexo, raza, cultura o habitat. Las repercusiones nocivas del abuso de drogas van desde el ámbito personal y familiar hasta el social, con sus secuelas en el deterioro de la salud, las relaciones interpersonales, el incremento de la marginalidad, la violencia y delitos múltiples. Se considera que el consumo prolongado de drogas determina relevantes daños biológicos, psicológicos, sociales y en la espiritualidad del consumidor. ${ }^{1}$

En la sociedad contemporánea, el consumo recreativo de alcohol se ha extendido por todos los ámbitos sociales y es aceptado en muchas culturas, como una costumbre integradora y festiva. ${ }^{2}$ Pero con el incremento del consumo de alcohol ha surgido también un complejo problema de salud pública, la dependencia y la adicción al alcohol, una enfermedad incurable, progresiva y mortal conocida como alcoholismo. ${ }^{2}$ Alejarse del consumo es un verdadero reto para los alcohólicos en rehabilitación toda vez que se reconoce la existencia de una baja efectividad del afrontamiento a las situaciones precipitantes de violación de abstinencia. ${ }^{3}$

Las adicciones se consideran una enfermedad crónica no transmisible, ya que los afectados sufren de consecuencias irreversibles a lo largo de sus vidas y no existe un vector físico, un germen animado que las transmita de un ser humano a otro. La lucha internacional contra el cultivo y comercialización de las drogas no ha logrado éxitos significativos. Apenas iniciado el actual milenio la comunidad internacional se percató de que las toxicomanías se habían convertido en un problema de salud de 
repercusión social que se ubicaba entre los tres grandes flagelos de la humanidad, junto con las guerras y las hambrunas. ${ }^{4}$

La proyección preventiva de los sistemas de salud plantea la urgente necesidad de actualizar y calificar a los profesionales y técnicos del equipo de salud para la atención específica de las conductas adictivas, la formación de promotores de salud para el problema de las adicciones y la incorporación del modelo de intervención psicosocial en contextos tradicionalmente clínicos.

La educación en materia de adicciones debe dirigirse a los individuos, las familias y la comunidad, sin obviar a los profesionales de la salud en formación pero la inclusión de dicha temática en los currículum de pre y posgrado ha sido hasta el momento muy limitada y su abordaje integral y sistémico requiere un decisivo impulso en los momentos actuales. ${ }^{5}$

A la Psicología de la Salud le corresponde valorar y promover el desarrollo de una concepción del mundo sólidamente sustentada, disponer de procesos autorreferenciales estables y realistas, lograr una motivación profesional coherente en el desempeño de cada cual y una sólida formación de valores y de proyectos de vida enriquecedores desde el punto de vista espiritual; resultados estos de trascendental importancia para desarrollar fortalezas e impedir que el flagelo de las drogas se apodere de las personas, los enajene y destruya. ${ }^{6}$ Por esa razón se hace imprescindible dotar a los profesionales con los recursos más efectivos para lograr los mejores resultados en su diario quehacer.

En América Latina, la expectativa de vida de la población adulta se ve reducida por el abuso de sustancias que dañan seriamente la conciencia, con el agravante de la elevada frecuencia de organicidad cerebral existente y el subsiguiente aumento de la vulnerabilidad ante los tóxicos. En la República de Ecuador la atención a pacientes consumidores de sustancias psicoactivas se ha desarrollado tradicionalmente desde la instancia privada. El estado ha seguido como línea de acción fundamental las actividades represivas contra el narcotráfico, aunque se reconoce el apoyo a algunos enfoques de tratamiento que han funcionado como unidad de farmacodependencia en algunos hospitales públicos (Universidad de Guayaquil-Consejo Nacional de Control de Sustancias Estupefacientes y Psicotrópicas. Proyecto Mejoramiento de la salud integral en poblaciones vulnerables al consumo de drogas, Guayaquil, 2010).

En la actualidad se hacen esfuerzos para controlar los programas de tratamientos públicos y privados ya que existen muchos centros que trabajan la rehabilitación del adicto al margen de las políticas establecidas e incluso que no cuentan con equipos profesionales interdisciplinarios. El Consejo Nacional de Control de Sustancias Estupefacientes y Psicotrópicas (CONSEP) registra un incremento significativo de la cantidad de personas que solicita atención por el método tradicional residencial (con internamiento), mientras los servicios ambulatorios, más cercanos al enfoque preventivo, pierden credibilidad.

Paralelamente a esta situación se notifica una disminución constante de la edad de inicio del consumo de drogas que en el 2008 se situó en los 12,3 años como promedio. El inicio temprano en el consumo de alcohol, ${ }^{7}$ y de otras drogas demuestran que los esfuerzos por parte de los diferentes sistemas sociales en cuanto a la prevención no han dado los resultados esperados y que el incremento de una cultura alcohólica propicia el consumo de esta droga socialmente aceptada como puerta de entrada a otras, lo que genera un alto costo psicológico y social a través de la violencia. 
El consumo de sustancias psicoactivas en Ecuador ha devenido en un problema de salud que debe ser atendido por las políticas públicas, por lo que las instituciones educativas, sociales y de salud propiamente dichas han sido llamadas a dar respuestas a la población involucrada. En este contexto, la Universidad Estatal de Guayaquil a través de la Facultad de Ciencias Psicológicas, presentó una propuesta pertinente e innovadora para contribuir a las políticas estatales de la salud con un enfoque integral teniendo en cuenta los enfoques de complejidad, diversidad y de género [Facultad de Ciencias Psicológicas Universidad de Guayaquil. Presentación del Centro de Docencia e Investigación para el Desarrollo Humano e el Buen Vivir (CDID). Guayaquil, 2010].

Con ello se pretende desarrollar modelos de intervención en la salud psicológica de la población con limitado acceso a estos servicios, ya sea por desconocimiento de su existencia o por sus elevados costos en el sector privado.

En el 2010 se presenta el proyecto "Mejoramiento de la salud integral en poblaciones vulnerables al consumo de drogas. Guayaquil, 2010" y se crea el CDID, destinado a convertirse en un centro de referencia de la salud mental en alianza, para la prevención y tratamiento, con instituciones públicas y privadas. En el 2011 se recabó la asesoría de expertos de la Unidad de Desarrollo Científico Tecnológica "Centro de Desarrollo Académico en Drogodependencias de la Universidad de Ciencias Médicas de La Habana" (CEDRO- UCMH), ${ }^{8}$ en virtud del insuficiente aprovechamiento de dichos recursos por parte de la comunidad, de ahí que la presente investigación se propusiera identificar la percepción comunitaria e institucional sobre las adicciones.

\section{MÉTODOS}

Se utilizó un diseño etnográfico. Los informantes clave fueron escogidos mediante muestreo intencional. Se aplicaron las siguientes técnicas:

\section{Análisis de documentos:}

- Proyecto: Mejoramiento de la salud integral en poblaciones vulnerables al consumo de drogas. Guayaquil, 2010. Universidad de Guayaquil. Consejo Nacional de Control de Sustancias Estupefacientes y Psicotrópicas.

- Presentación del CDID. Guayaquil, 2010. Facultad de Ciencias PsicológicasUniversidad de Guayaquil.

- Programa de la asignatura Psicología de la Salud. Carrera de Psicología Clínica. Facultad de Ciencias Psicológicas-Universidad de Guayaquil 2010.

- Programa de la asignatura Farmacodependencias. Carrera de Psicología Clínica. Facultad de Ciencias Psicológica-Universidad de Guayaquil 2010.

- Texto básico de la asignatura Farmacodependencias. (García Jaime J. Tratado sobre Drogas Psicoactivas IV Edición actualizada, Guayaquil, 2009). 
2. Observación participante:

- Sesiones de trabajo e intercambio en el CDID.

- Sesiones de trabajo e intercambio en la Facultad de Ciencias Psicológicas de la Universidad de Guayaquil.

3. Entrevista en profundidad:

- Dirección y coordinación académica de la Facultad.

- Dirección ejecutiva y vicedirección técnica del CDID.

- Psicólogos y otros profesionales.

- Operadores vivenciales.

- Trabajadores y usuarios del Centro.

4. Entrevista grupal focalizada:

- Actores sociales de la comunidad.

- Docentes de la Facultad de Ciencias Psicológicas de la Universidad de Guayaquil.

- Profesionales interesados.

- Directivos del CDID.

El procesamiento de la información se realizó por conducto de un análisis de contenido que se estructuró en la siguiente secuencia: reducción de los parlamentos de los informantes clave a proposiciones, segmentación de las proposiciones en categorías, devolución del entramado de categorías a los informantes clave para su validación, refinamiento de las categorías, codificación y vínculo intercategorías. (Díaz Llanes G. El análisis cualitativo de los datos. En: Programa de Maestría en Psicología de la Salud. La Habana. CEDISAP. 2006).

\section{RESULTADOS}

El análisis de contenido permitió identificar percepciones sobre el tema adicciones, desde las siguientes dimensiones.

Gestión:

- Desestimación por parte de directivos de organizaciones de la incidencia de las conductas adictivas en el ambiente laboral y sus afectaciones a la bioseguridad. 
- Desconocimiento en las comunidades aledañas acerca de los servicios que brinda el Centro y sus posibilidades de acceso.

- Inconstancia en las iniciativas para promover la intersectorialidad y la colaboración institucional en el abordaje de las conductas adictivas.

- Deficiente gestión para la búsqueda de alternativas que garanticen el autofinanciamiento y el desarrollo organizacional de la institución.

\section{Docente-Asistencial:}

- Insuficientes oportunidades prácticas en la formación de pregrado para el desarrollo de competencias para la prevención y atención de las conductas adictivas.

- Carencia de formación especializada y de posgrado referida a la prevención y atención de las adicciones.

- Escasa credibilidad profesional de la modalidad ambulatoria de tratamiento para la deshabituación de adictos.

Investigativa:

- Ausencia de estrategias y líneas de investigación psicosocial y comunitaria que aborden multilateralmente el problema de las drogodependencias.

- Limitada preparación en aspectos metodológicos e instrumentales y en gestión de proyectos de investigación sobre adicciones.

- Insuficientes proyectos de investigación que aborden el componente psicosocial de la drogodependencia en la comunidad.

\section{DISCUSIÓN}

Los documentos evaluados mostraron aspectos contradictorios. Mientras la propuesta de CDID privilegia la necesidad de instaurar un modo de actuar preventivo congruente con el modelo social expansivo que demanda el problema a abordar, los programas y textos de las asignaturas comprometidas con la formación de los psicólogos que pudieran brindar servicios en dicha institución no aportan herramientas suficientes para el tipo de abordaje que se pretende.

Por ejemplo, aun cuando el objetivo pedagógico declarado en el programa vigente de la asignatura Psicología de la Salud es "Conocer la evolución histórica que ha experimentado la Psicología de la Salud, así como su posicionamiento, dentro del Sistema Nacional de Salud; identificando en el proceso las diferentes áreas y zonas de solapamiento, para el surgimiento y desarrollo de ella como disciplina científica", no se concretan modos de actuar propios concibiéndose solo como una ampliación del perfil o acciones de intervención del psicólogo clínico. 
A partir de ello en el estudio desarrollado se sugirió la necesidad de lograr un verdadero posicionamiento del modelo psicosocial en el abordaje de las conductas adictivas. Para contribuir a ello se ejecutaron talleres introductorios al tema con vistas a demostrar la pertinencia del abordaje ambulatorio.

Una de las mayores preocupaciones de la dirección de la facultad y del CDID es la escasa aceptación de los servicios ambulatorios en las comunidades aledañas a pesar de que se brindan a costos simbólicos. EI CDID está rodeado de 35 comunidades de los sectores Prosperita, Pájaro azul, Martha de Roldós, Sauces, Mapasingue Este y Mapasingue Oeste. Se trata de una población humilde conformada por asentamientos de personas emigrantes de otras regiones del país (Manabí, Azuay, Tungurahua, Los Ríos) que se dedican a actividades como la construcción, la artesanía y el comercio informal. El nivel de instrucción predominante es el correspondiente a la enseñanza primaria con un porcentaje elevado de deserción escolar, debido a los embarazos de las adolescentes, migración y trabajo infantil. Los servicios de salud son insuficientes frente a la demanda de la población. El nivel de seguridad ciudadana es mínimo ya que existe una elevada presencia de tráfico y consumo de drogas, pandillas juveniles, violencia, entre otros.

Es común que los vecinos prefieran internar a sus familiares adictos porque confían en un tratamiento que al menos temporalmente los libera de la responsabilidad de atenderlos. En general, los servicios residenciales o con internamiento son más frecuentemente utilizados por las comunidades terapéuticas y por los modelos médicos de intervención. En estos casos se considera al individuo dependiente como una persona incapaz de estar en su medio (Díaz Llanes G. El análisis cualitativo de los datos. En: Programa de Maestría en Psicología de la Salud. La Habana. CEDISAP. 2006.), y por lo tanto, es necesario mantenerlo bajo el control de otras personas constantemente. Hay formas de internamiento de corta estancia, que tienen como fin ofrecer apoyo al paciente y su familia durante el período más crítico.

Sin embargo, los tratamientos ambulatorios ofrecen un espacio de intervención que incluye la casa y el trabajo del paciente, lugares que ellos tienen que aprender a manejar. La permanencia en esta modalidad fluctúa desde algunas horas a programas de medio día o de hospitalización parcial (hospital de día). Dadas las características de las intervenciones ambulatorias, estas son de bajo costo y de fácil acceso, ya que las personas no se ven obligadas a internarse por períodos prolongados ni a interrumpir sus trabajos o estudios. ${ }^{9}$

Además, el paciente está en permanente interacción con el medio y su familia (si la hubiere), características que hacen de las intervenciones ambulatorias un espacio que incluye no solo las estrategias de tratamiento propiamente, sino que también debe dar respuesta a las contingencias que se originan de las relaciones que el paciente establece con su medio y su familia. En general, se plantea que en los servicios ambulatorios los usuarios suelen valorar mejor la información sobre su tratamiento, reconocen la eficacia del equipo terapéutico en sus tareas y el hecho de haber recibido la ayuda necesaria para la búsqueda de soluciones, a su vez, el equipo tiene mayores posibilidades de diálogo con el paciente. ${ }^{10}$

Desde esta perspectiva, el tratamiento ambulatorio facilita que el individuo aprenda habilidades para desempeñarse en el mundo en que se desenvuelve habitualmente y por tanto su aplicación es consecuente con el esfuerzo que se realiza para la implementación de estrategias aplicables a la atención primaria de salud y a desarrollar la iniciativa sobre Redes Integradas de Servicios de Salud propuesta por 
OPS/OMS como alternativa ante el desafío de la fragmentación presente en el contexto latinoamericano. ${ }^{11}$

En el análisis de la información aportada se constataron propuestas que incluyen promover el trabajo preventivo a partir de incorporar los educadores de los colegios del área y de acercarse a los lideres informales de las comunidades para promover mayor aceptación de los servicios.

Otro de los aspectos que emergió del análisis fue la necesidad de articular las asignaturas del pregrado con el CDID de manera tal que los estudiantes puedan desarrollar competencias para la prevención y atención de las conductas adictivas desde las perspectivas de la Psicología Clínica y de la Salud, la Psicología Educativa, la Psicología Organizacional y otras. Además, se propuso la actualización del programa de la asignatura Farmacodependencias de manera tal que además de contemplar las clasificaciones y efectos fundamentales del consumo de sustancias psicoactivas, se contemplen modelos psicológicos explicativos y de intervención.

Se consideró pertinente desarrollar un programa de posgrado sobre el abordaje integral de las conductas adictivas. La propuesta pudiera ser ejecutada en el propio CDID contribuyendo a la capacitación de los recursos humanos y al autofinanciamiento de la institución. Además, se propuso la impartición de cursos dirigidos a otras instituciones que aportarían usuarios a los servicios del CDID. En ese sentido, se destaca un curso de capacitación en el abordaje de conductas de riesgo en los adolescentes dirigido a los orientadores de los colegios de las comunidades aledañas. También se propone la creación de una consultoría especializada en la incidencia de las conductas adictivas en el ambiente laboral que aborde sus afectaciones a la bioseguridad.

Finalmente, se constató la necesidad de desarrollar una estrategia de investigación psicosocial y comunitaria que proporcione salida a los proyectos de los estudiantes de pre y posgrado. Se propone la capacitación y asesoría en aspectos metodológicos e instrumentales y en gestión de proyectos comunitarios vinculados al problema de las conductas adictivas. Como parte de ello se propuso desarrollar un plan de difusión de los servicios que brinda el CDID en las comunidades aledañas utilizando los colegios como mediadores interesados y divulgar líneas de investigaciones coherentes con la misión y visión del centro entre estudiantes y docentes que deseen desarrollar competencias para la investigación.

Como resultado de la investigación desarrollada se elaboró el proyecto "Asesoramiento académico para el desarrollo del abordaje integral y la valoración psicosocial de las conductas adictivas", en el que se incluye la propuesta de creación de una consultoría docente e investigativa sobre conductas adictivas y la presentación del programa de posgrado "Maestría en abordaje integral de las conductas adictivas". Los beneficiarios directos de estas propuestas serían:

- Profesionales que trabajan en la prevención y atención de las conductas adictivas y que necesiten de asesoría y capacitación de posgrado.

- Estudiantes de la Universidad de Guayaquil que requieran formación de pregrado sobre el abordaje integral de las conductas adictivas.

- Orientadores educativos y actores sociales de las comunidades con riesgo adictivo y de violencia social. 
- Organizaciones educativas y laborales que necesiten afrontar el problema de la bioseguridad y otros aspectos relacionados con las conductas adictivas.

- Personas sanas, con riesgo, con enfermedad adictiva o rehabilitada, que accedan al servicio de consultoría.

La percepción comunitaria e institucional de las adicciones, emergente del vínculo de perspectivas de gestión comunitaria, docente-asistenciales e investigativas, revela una marcada restricción al modelo clínico-asistencial, que hace necesario el diseño e implementación de un proyecto a esos niveles que facilite el posicionamiento del modelo salubrista.

\section{REFERENCI AS BI BLI OGRÁFICAS}

1. González R. Clínica Psiquiátrica Actual. La Habana: Editorial Félix Varela; 2006.

2. González R, Galán G. El alcohol: la droga bajo la piel del cordero. Rev Hosp Psiquiátrico Habana [Internet]. 2007 [citado 10 Oct 2011];4(3). Disponible en: http://ucha. blogia.com/2008/041601-revista-del-hospital-psiquiatrico-de-lahabana.php

3. Iglesias S, Fabelo JR, Artiles E. Percepción de efectividad del afrontamiento a situaciones precipitantes de violación de abstinencia en alcohólicos. inFÁRMAte. 2011; 7(26): e075-092.

4. Organización de las Naciones Unidas. Informe sobre tendencias mundiales de las drogas ilícitas. New York: ONU; 2003.

5. González R. Mensaje a multiplicadores comunitarios sobre la repercusión social global del uso indebido de drogas. Rev Cubana Salud Pública [Internet]. 2010 [citado $10 \quad$ Oct 2011 ];36(1). Disponible en: http://scielo.sld.cu/scielo.php?script=sci_arttext\&pid=S0864$\underline{34662010000100016 \& \operatorname{lng}=\mathrm{es} \& \mathrm{nrm}=\mathrm{iso \& t} \operatorname{lng}=\mathrm{es}}$

6. Fabelo JR, Iglesias S, Bravo V. Circunstancias potenciales de riesgo adictivo en niños, adolescentes y jóvenes. inFÁRMAte. 2011;(26): e001-011.

7. González R Significación médico social y ético-humanística de las drogas. Rev Cubana Salud Pública [Internet]. 2007 [citado 10 Oct 2011];33(1). Disponible en: http://scielo.sld.cu/scielo.php?script=sci_arttext\&pid=S086434662007000100002\&lng=es

8. Gálvez E. CEDRO: Antídoto de las drogas en la Universidad Médica. Rev Hab Ciencias Médicas. 2005;4(4).

9. Arcila MR, Del Solar G. Osorio D. Villarino AM. La dependencia al consumo de drogas. Una aproximación teórico práctica al tratamiento ambulatorio. Santiago. Dolmen. 1999. 
10. Rodríguez M, Jiménez Lerma J M, Iraurgi I. Murua F, Bacigalupe L, Chavarri MR, Balsategi B. Evaluación de la Satisfacción con el Tratamiento en un Centro Ambulatorio de Drogodependencias a través del Treatment Perceptions Questionnaire (TPQ). Adicciones. 2002; 14(4):417-24.

11. López P, Alonso L, García AJ, Serrano AC, Ramírez LB. Metodología para la caracterización de una red de servicios de salud y su aplicación en el contexto ecuatoriano. Rev Cubana Salud Pública [Internet]. 2011 [citado 10 Oct 2011];37(2):130-6.

Recibido: 14 de octubre de 2011.

Aprobado: 21 de octubre de 2011.

Justo Reinaldo Fabelo Roche. Calle 66 No. 2715 e/ 27 y 29. San Antonio de los Baños 32500. Artemisa. Cuba.

Teléf.: 047 383865. Correo electrónico: fabelo@infomed.sld.cu 